\title{
FUNCTIONS WITH A DENSE SET OF PROPER LOCAL MAXIMUM POINTS
}

\author{
ALFONSO VILLANI ${ }^{1}$
}

\begin{abstract}
Let $X$ be any metric space. The existence of continuous real functions on $X$, with a dense set of proper local maximum points, is shown. Indeed, given any $\sigma$-discrete set $S \subset X$, the set of all $f \in C(X)$, which assume a proper local maximum at each point of $S$, is a dense subset of $C(X)$. This implies, for a perfect metric space $X$, the density in $C(X, Y)$ of "nowhere constant" continuous functions from $X$ to a normed space $Y$. In this way, two questions raised in [2] are solved.
\end{abstract}

The existence of continuous functions $f: \mathbf{R} \rightarrow \mathbf{R}$ having some proper local maximum point within each open subset of $\mathbf{R}$ is well known. A nice construction of such a function is given in [1]. In this note we show that continuous real functions with this property do exist on any metric space $X$. Indeed, we prove (Theorem 1 ) that for every $\sigma$-discrete set $S \subset X$, the set of continuous real functions on $X$, which have a proper local maximum at each point of $S$, is dense in $C(X)$, endowed with a certain topology which, in general, is strictly finer than that of uniform convergence; in particular, functions with a dense set of proper local maximum points are dense in $C(X)$. As a corollary, if $X$ is a perfect metric space, we get the density in $C(X, Y)$ of nowhere constant continuous functions from $X$ to a normed space $Y$. This answers two questions recently raised in [2] and enables us to improve some results established there.

Throughout, $X$ is a metric space with metric $d, Y$ is a normed space with norm \|\| , and $C(X, Y)$ denotes the set of all continuous functions $f: X \rightarrow Y$. When $Y=\mathbf{R}$ we put $C(X)=C(X, Y)$. We denote by $\tau$ the topology on $C(X, Y)$ in which basic neighbourhoods of $f \in C(X, Y)$ are the sets

$$
\{g \in C(X, Y) \mid\|g(x)-f(x)\|<\varepsilon(x) \text { for each } x \in X\}
$$

with $\varepsilon \in C(X), \varepsilon>0$ everywhere in $X$. It is clear that $\tau$ is stronger than the topology $v$ induced by the metric $\rho$ of uniform convergence, i.e.,

$$
\rho(f, g)=\min \{1, \sup \{\|f(x)-g(x)\| \mid x \in X\}\} .
$$

Moreover, it is easy to realize that $\tau$ and $v$ coincide if and only if $X$ is compact.

Received by the editors January 18, 1984 and, in revised form, July 20, 1984.

1980 Mathematics Subject Classification. Primary 54C30; Secondary 54E35.

${ }^{1}$ Research partially supported by a national project of the Italian Ministero della Pubblica Istruzione and by G.N.A.F.A. of Italian Consiglio Nazionale delle Ricerche. 
For subsets of $X$ the term discrete is used here in the following sense: a set $D \subset X$ is said to be discrete if $D$ has no accumulation points. A $\sigma$-discrete set is a countable union of discrete sets. For collections $\mathscr{F}$ of subsets of $X$ our terminology is standard: $\mathscr{F}$ is said to be discrete if every point $x \in X$ has a neighbourhood meeting at most one set in $\mathscr{F}$. A $\sigma$-discrete collection is a countable union of discrete collections.

Since the definition of discrete set given here is nonstandard, some comments are necessary. Obviously a discrete set as defined here is also discrete in the usual sense, i.e., its relative topology is the discrete topology. The converse is not true (e.g., $X=\mathbf{R}, D=\{1 / n \mid n=1,2, \ldots\})$. Nevertheless, if a set $D$ is discrete in the usual sense, then it is also $\sigma$-discrete according to our definition. Indeed, for each $\rho>0$, the set $D_{\rho}=\{x \in D \mid d(x, D-\{x\}) \geqslant \rho\}$ has no accumulation points; hence $D=$ $\bigcup_{\rho>0} D_{\rho}=\cup_{n=1}^{\infty} D_{1 / n}$ is $\sigma$-discrete in our sense. It follows that, in a metric space, the definition of $\sigma$-discrete set given here and the usual one are equivalent. This is no longer true in a general topological space. We do not give any explicit proof of the last remark. However, a counterexample can easily be constructed by the interested reader in the space of all real functions on $\mathbf{R}$ endowed with the topology of pointwise convergence.

Given a function $f: X \rightarrow \mathbf{R}$, we say that $x \in X$ is a proper local maximum point for $f$ if $f(U-\{x\}) \subset(-\infty, f(x))$ for some neighbourhood $U$ of $x$. The set of all proper local maximum points for $f$ is denoted by $M(f)$. Note that for every $t>0$, the set

$$
M_{t}(f):=\{x \in X \mid 0<d(z, x)<t \Rightarrow f(z)<f(x)\}
$$

is discrete; hence, the set

$$
M(f)=\bigcup_{t>0} M_{t}(f)=\bigcup_{n=1}^{\infty} M_{1 / n}(f)
$$

is $\sigma$-discrete. Conversely, Theorem 1 shows that for any $\sigma$-discrete set $S \subset X$ there is always a continuous function $f: X \rightarrow \mathbf{R}$ such that $M(f) \supset S$.

For the reader's convenience we state a result from [2] that will be used in the sequel. By $\mathscr{R}(X, Y)$ we denote the set of all $f \in C(X, Y)$ which are nowhere constant (locally nonconstant according to the terminology of [2]), i.e., such that int $f^{-1}(y)=$ $\varnothing$ for all $y \in Y$. When $Y=\mathbf{R}$ we put $\mathscr{R}(X)=\mathscr{R}(X, Y)$. The convex hull of a set $W \subset Y$ is denoted by $\operatorname{conv}(W)$.

TheOrem A ([2, TheOREM 2.1]). Suppose $X$ is locally connected and $\mathscr{R}(X)=\varnothing$. Then, for every function $f \in C(X, Y)$ and every positive constant $\varepsilon$, there exists a function $g \in \mathscr{R}(X, Y)$ such that $\rho(g, f) \leqslant \varepsilon$. Moreover, $g(X) \subset \operatorname{conv}(f(X))$ provided that $f$ is not constant.

To begin, we prove two lemmas.

Lemma 1. Let $D \subset X$ be a nonempty discrete set. Then there exists a discrete collection $\left\{B_{s} \mid s \in D\right\}$ of closed balls with each $B_{s}$ centered at $s$. 
Proof. If $D$ is a finite set, then any collection of pairwise disjoint closed balls, with centers at points of $D$, is discrete. This is no longer true, in general, if the set $D$ is infinite. Then we proceed as follows.

For each $x \in X$ let $d_{x}$ denote the positive number $d(x, D-\{x\})$. Also, fix any $\gamma \in\left(0, \frac{1}{2}\right)$, and denote by $B_{s}, s \in D$, the closed ball centered at $s$ with radius $r_{s}:=\gamma d_{s}$. Then $\left\{B_{s} \mid s \in D\right\}$ is a discrete collection. Indeed, we claim that for every $x \in X$, any ball $C_{x}$ with center at $x$ and radius $\rho_{x} \leqslant\left(\frac{1}{2}-\gamma\right) d_{x}$ meets at most one ball $B_{s}$. This is proved by contradiction as follows. Assume that $C_{x} \cap B_{s_{1}}$ and $C_{x} \cap B_{s_{2}}$ are nonempty for some $x \in X, s_{1}, s_{2} \in D, s_{1} \neq s_{2}$, and let $z_{i} \in C_{x} \cap B_{s_{i}}$, $i=1,2$. Then $d_{x} \geqslant \max \left\{d_{s_{1}}, d_{s_{2}}\right\}$, for otherwise, assuming for instance $d_{s_{1}}>d_{x}$, $d_{s_{1}} \geqslant d_{s_{2}}$, we would get the contradiction

$$
\begin{aligned}
d_{s_{1}} & \leqslant d\left(s_{1}, s_{2}\right) \leqslant d\left(s_{1}, z_{1}\right)+d\left(z_{1}, z_{2}\right)+d\left(z_{2}, s_{2}\right) \\
& \leqslant r_{s_{1}}+2 \rho_{x}+r_{s_{2}} \leqslant \gamma d_{s_{1}}+(1-2 \gamma) d_{x}+\gamma d_{s_{2}} \\
& <\gamma d_{s_{1}}+(1-2 \gamma) d_{s_{1}}+\gamma d_{s_{1}}=d_{s_{1}} .
\end{aligned}
$$

The contradiction

$$
\begin{aligned}
d_{x} & \leqslant d\left(x, s_{1}\right) \leqslant d\left(x, z_{1}\right)+d\left(z_{1}, s_{1}\right) \leqslant \rho_{x}+r_{s_{1}} \\
& \leqslant\left(\frac{1}{2}-\gamma\right) d_{x}+\gamma d_{s_{1}} \leqslant\left(\frac{1}{2}-\gamma\right) d_{x}+\gamma d_{x}=d_{x} / 2
\end{aligned}
$$

follows, assuming, for instance, $s_{1} \neq x$, and the lemma is proved.

Lemma 2. Let $\varphi, \eta \in C(X)$ with $\eta>0$ everywhere in $X$. Also, let $D \subset X$ be a nonempty discrete set and $H$ a closed set with $D \cap H=\varnothing$. Then there exist $\psi \in C(X)$ and $\left\{B_{s} \mid s \in D\right\}$, a discrete collection of closed balls with each $B_{s}$ centered at $s$, such that

(i) $\left(\cup_{s \in D} B_{s}\right) \cap H=\varnothing$,

(ii) $\psi=\varphi$ in $X-\bigcup_{s \in D} B_{s}$,

(iii) $\varphi \leqslant \psi<\varphi+\eta$ everywhere in $X$, and

(iv) $\psi(x)<\psi(s)$ for every $s \in D$ and $x \in B_{s}-\{s\}$.

Proof. By Lemma 1 it is possible to associate a positive number $r_{s}$ with each $s \in D$ in such a way that the collection of balls $B_{s}$, with center at $s \in D$ and radius $r_{s}$, is discrete. Also, it is clearly possible, decreasing the numbers $r_{s}$ if necessary, to fulfill condition (i). In the same way, owing to the continuity of $\varphi$ and $\eta$, it can be assumed that, for every $s \in D$, the inequalities

$$
\varphi(x)<\varphi(s)+\frac{1}{2} \eta(s)<\varphi(x)+\eta(x)
$$

hold at each point $x$ of $B_{s}$.

Now define $\psi: X \rightarrow \mathbf{R}$ as follows:

$$
\begin{gathered}
\psi(x)=\varphi(x) \quad \text { if } x \in X-\bigcup_{s \in D} B_{s}, \\
\psi(x)=\left(1-r_{s}^{-1} d(x, s)\right)\left(\varphi(s)+\frac{1}{2} \eta(s)\right)+r_{s}^{-1} d(x, s) \varphi(x) \quad \text { if } x \in B_{s}, s \in D .
\end{gathered}
$$


Then, by the discreteness of $\left\{B_{s} \mid s \in D\right\}, \psi$ is well defined and continuous. Also, by definition, (ii) is satisfied. Finally, having in mind the inequalities (*), it is an easy matter to check the validity of conditions (iii) and (iv). The lemma is therefore proved.

Now we prove our main theorem.

TheOREM 1. Let $f, \varepsilon \in C(X)$ with $\varepsilon>0$ everywhere in $X$. Also let $S \subset X$ be a nonempty $\sigma$-discrete set and $K \subset X$ a closed set with $S \cap K=\varnothing$. Then there exists $g \in C(X)$ such that

(i) $\left.g\right|_{K}=\left.f\right|_{K}$,

(ii) $f \leqslant g<f+\varepsilon$ everywhere in $X$, and

(iii) $M(g) \supset S$.

ProOF. If $S$ is a discrete set, the existence of such a function $g$ is guaranteed by Lemma 2. Hence, assume that $S$ is not discrete. Then we have $S=\cup_{n=0}^{\infty} D_{n}$, with $D_{0}$, $D_{1}, \ldots$ pairwise disjoint nonempty discrete sets. We set $E_{n}=\bigcup_{k=0}^{n} D_{k}, n=0,1, \ldots$

We start by applying Lemma 2 with $\varphi=f, \eta=\varepsilon / 2, D=D_{0}$, and $H=K$. Accordingly, there exist $f_{0} \in C(X)$ and $\left\{B_{s} \mid s \in D_{0}\right\}$, a collection of closed balls with each $B_{s}$ centered at $s$, such that

$(1)_{0}\left\{B_{s} \mid s \in D_{0}\right\}$ is a discrete collection of sets,

(2) ${ }_{0}\left(\cup_{s \in D_{0}} B_{s}\right) \cap K=\varnothing$,

(3) $0=f_{0}$ in $X-\cup_{s \in D_{0}} B_{s}$,

(4) ${ }_{0} f \leqslant f_{0}<f+\varepsilon / 2$ everywhere in $X$,

$(5)_{0} f_{0}(x)<f_{0}(s)$ for every $s \in D_{0}$ and $x \in B_{s}-\{s\}$.

Next we show by induction that there exist $\left\{f_{n} \mid n=1,2, \ldots\right\}$, a sequence in $C(X)$, and $\left\{B_{s} \mid s \in \bigcup_{n=1}^{\infty} D_{n}\right\}$, a collection of closed balls with $B_{s}$ centered at $s$, such that the following conditions are satisfied for every $n=1,2, \ldots$ :

(1) ${ }_{n}\left\{B_{s} \mid s \in D_{n}\right\}$ is a discrete collection of sets,

(2) ${ }_{n}\left(\cup_{s \in D_{n}} B_{s}\right) \cap\left(K \cup E_{n-1}\right)=\varnothing$,

(3) $f_{n}=f_{n-1}$ in $X-\bigcup_{s \in D_{n}} B_{s}$,

(4) $f_{n-1} \leqslant f_{n}<f_{n-1}+\varepsilon / 2^{n+1}$ everywhere in $X$,

(5) ${ }_{n} f_{n}(x)<f_{n}(s)$ for every $s \in E_{n}$ and $x \in B_{s}-\{s\}$,

(6) ${ }_{n} s \in D_{n}, t \in E_{n-1}, B_{s} \cap B_{t} \neq \varnothing$ imply $s \in B_{t}$.

To prove this, let us assume that functions $f_{0}, \ldots, f_{n}$ and balls $\left\{B_{s} \mid s \in E_{n}\right\}$ have been found in such a way that conditions $(1)_{k}-(5)_{k}, k=0, \ldots, n$, and, if $n>0$, also $(6)_{k}, k=1, \ldots, n$, are satisfied, and construct $f_{n+1}$ and $\left\{B_{s} \mid s \in D_{n+1}\right\}$ such that $(1)_{n+1}-(6)_{n+1}$ hold.

Decompose $D_{n+1}$ as $D_{n+1}=D_{n+1}^{\prime} \cup D_{n+1}^{\prime \prime}$, with $D_{n+1}^{\prime}=D_{n+1}-\cup_{t \in E_{n}} B_{t}$ and $D_{n+1}^{\prime \prime}=D_{n+1}-D_{n+1}^{\prime}$.

If $D_{n+1}^{\prime}=\varnothing$ we introduce $h$ by setting $h=f_{n}$.

If $D_{n+1}^{\prime} \neq \varnothing$ we apply Lemma 2 again, with $\varphi=f_{n}, \eta=\varepsilon / 2^{n+3}, D=D_{n+1}^{\prime}$, and $H=K \cup\left(\cup_{t \in E_{n}} B_{t}\right)\left(H\right.$ is a closed set by assumptions $\left.(1)_{k}, k=0, \ldots, n\right)$. Then there exist $h \in C(X)$ and $\left\{B_{s} \mid s \in D_{n+1}^{\prime}\right\}$, a collection of closed balls with each $B_{s}$ centered at $s$, such that

(a) $\left\{B_{s} \mid s \in D_{n+1}^{\prime}\right\}$ is a discrete collection of sets,

(b) $\left(\cup_{s \in D_{n+1}^{\prime}} B_{s}\right) \cap\left(K \cup\left(\cup_{t \in E_{n}} B_{t}\right)\right)=\varnothing$, 
(c) $h=f_{n}$ in $X-\bigcup_{s \in D_{n+1}^{\prime}} B_{s}$,

(d) $f_{n} \leqslant h<f_{n}+\varepsilon / 2^{n+3}$ everywhere in $X$,

(e) $h(x)<h(s)$ for every $s \in D_{n+1}^{\prime}$ and $x \in B_{s}-\{s\}$.

Now, consider $D_{n+1}^{\prime \prime}$. If $D_{n+1}^{\prime \prime}=\varnothing$, then the collection $\left\{B_{s} \mid s \in D_{n+1}\right\}$ has already been defined; moreover, letting $f_{n+1}=h$, it is clear, by (a)-(e) and (5) $)_{n}$, that conditions $(1)_{n+1}-(6)_{n+1}$ are fulfilled.

Hence, suppose that $D_{n+1}^{\prime \prime} \neq \varnothing$ and, according to Lemma 1, let $\left\{U_{s} \mid s \in D_{n+1}^{\prime \prime}\right\}$ be any discrete collection of open balls, with each $U_{s}$ centered at $s$. Denote by $\Delta_{s}$, for each $s \in D_{n+1}^{\prime \prime}$, the set $\left\{t \in E_{n} \mid s \in B_{t}\right\}$. By $(1)_{k}, k=0, \ldots, n, \Delta_{s}$ contains at most $n+1$ elements. Let $\tau_{s}=\min \left\{f_{n}(t)-f_{n}(s) \mid t \in \Delta_{s}\right\}$. By (5) ${ }_{n}, \tau_{s}$ is a positive number. Also denote by $C_{s}$ the union of those balls $B_{t}, t \in E_{n} \cup D_{n+1}^{\prime}$, which do not contain $s$. By $(1)_{k}, k=0, \ldots, n$, and (a) (if $\left.D_{n+1}^{\prime} \neq \varnothing\right), C_{s}$ is a closed set. Then apply Lemma 2 with $\varphi=h, \eta=\eta_{s}=\min \left\{\varepsilon / 2^{n+3}, \tau_{s}\right\}, D=\{s\}, H=K \cup C_{s} \cup \Delta_{s} \cup\left(X-U_{s}\right)$. Accordingly, there exist $h_{s} \in C(X)$ and $B_{s}$, a closed ball with center at $s$, such that $(\beta)_{s} B_{s} \subset U_{s}, B_{s} \cap\left(K \cup C_{s} \cup \Delta_{s}\right)=\varnothing$,

$(\gamma)_{s} h_{s}=h$ in $X-B_{s}$,

$(\delta)_{s} h \leqslant h_{s}<h+\eta_{s}$ everywhere in $X$,

$(\varepsilon)_{s} h_{s}(x)<h_{s}(s)$ for every $x \in B_{s}-\{s\}$.

Do this for each $s \in D_{n+1}^{\prime \prime}$. Then the collection $\left\{B_{s} \mid s \in D_{n+1}\right\}$ has been defined. Moreover, having in mind condition $(\beta)_{s}, s \in D_{n+1}^{\prime \prime}$, the discreteness of $\left\{U_{s} \mid s \in\right.$ $\left.D_{n+1}^{\prime \prime}\right\}$ and also, if $D_{n+1}^{\prime} \neq \varnothing$, conditions (a)-(b), it is clear that $(1)_{n+1},(2)_{n+1}$, and (6) ${ }_{n+1}$ are fulfilled. Let $f_{n+1}: X \rightarrow \mathbf{R}$ be defined as follows:

$$
\begin{aligned}
& f_{n+1}(x)=h(x) \text { if } x \in X-\bigcup_{s \in D_{n+1}^{\prime \prime}} B_{s}, \\
& f_{n+1}(x)=h_{s}(x) \text { if } x \in B_{s}, s \in D_{n+1}^{\prime \prime} .
\end{aligned}
$$

By $(1)_{n+1}, f_{n+1}$ is well defined; also, by $(1)_{n+1}$ and $(\gamma)_{s}, s \in D_{n+1}^{\prime \prime}$, it is a continuous function. Moreover, using conditions $(\delta)_{s}, s \in D_{n+1}^{\prime \prime}$, and, as usual, (c)-(d), if $D_{n+1}^{\prime} \neq \varnothing$, it is easy to check the validity of $(3)_{n+1}-(4)_{n+1}$. Let us show that also $(5)_{n+1}$ is satisfied. This will conclude the inductive argument. Let $s \in E_{n+1}, x \in B_{s}$ $-\{s\}$. First assume $s \in E_{n}$, so $f_{n+1}(s)=f_{n}(s)$ by $(2)_{n+1}-(3)_{n+1}$. If $x \notin \bigcup_{t \in D_{n+1}} B_{t}$, then $f_{n+1}(x)=f_{n}(x)<f_{n}(s)=f_{n+1}(s)$ by (3) ${ }_{n+1}$ and (5) ${ }_{n}$. If $x \in B_{t}$ for some $t \in D_{n+1}$, then $t \in B_{s}$ by $(6)_{n+1}$; hence, $t \in D_{n+1}^{\prime \prime}$ and $s \in \Delta_{t}$; it follows that, by $(\varepsilon)_{t}$, $(\delta)_{t}$, and also (b)-(c), if $D_{n+1}^{\prime} \neq \varnothing$, then

$$
\begin{aligned}
f_{n+1}(x) & =h_{t}(x) \leqslant h_{t}(t)<h(t)+\tau_{t} \\
& =f_{n}(t)+\tau_{t} \leqslant f_{n}(s)=f_{n+1}(s) .
\end{aligned}
$$

Now consider the case $s \in D_{n+1}$. Then we again get $f_{n+1}(x)<f_{n+1}(s)$, using condition (e) if $s \in D_{n+1}^{\prime}$ or condition $(\varepsilon)_{s}$ if $s \in D_{n+1}^{\prime \prime}$.

At this point we are in a position to define the function $g$ that we are looking for. Let $g(x)=\lim _{n} f_{n}(x)$ for every $x \in X$. By conditions $(4)_{n}, n=0,1,2, \ldots, g$ is a well-defined, continuous function satisfying (ii). Also, by $(2)_{n}-(3)_{n}, n=0,1, \ldots$, it is clear that (i) is fulfilled. Let us check (iii). To this end it is enough to show that 
$g(x)<g(s)$ for every $s \in S$ and $x \in B_{s}-\{s\}$. Let $s$ and $x$ be fixed as above. Suppose $s \in D_{\nu}$; then $f_{\nu}(s)=f_{\nu+1}(s)=\cdots=g(s)$ by $(2)_{n}-(3)_{n}, n=\nu+1, \nu+$ $2, \ldots$ Moreover, let

$$
L=\left\{n \mid x \in \bigcup_{t \in D_{n}} B_{t}\right\}
$$

so $\nu \in L$. We distinguish two cases, according as $\nu=\max L$ or otherwise. In the first case, by $(3)_{n}, n=\nu+1, \nu+2, \ldots$, and (5), we have $g(x)=f_{\nu}(x)<f_{\nu}(s)=g(s)$. In the second case, let $m^{*} \in L$ and $t \in D_{m^{*}}$ be fixed in such a way that $m^{*}>\nu$ and $x \in B_{t}$. Then, for every $m \geqslant m^{*}$, by $(5)_{m}$ and $(2)_{n}-(3)_{n}, n=m^{*}+1, m^{*}+2, \ldots$, we get $f_{m}(x) \leqslant f_{m}(t)=f_{m^{*}}(t)$. Consequently, by $(5)_{m^{*}}, g(x) \leqslant f_{m^{*}}(t)<f_{m^{*}}(s)=$ $g(s)$. This concludes the proof.

Let us point out that Theorem 1 implies that for every $\sigma$-discrete set $S \subset X$, the set $\mathscr{M}_{S}$ of all functions $f \in C(X)$, such that $M(f) \supset S$, is dense in $C(X)$ when the latter is endowed with the topology $\tau$. In particular, we get the following

COROLLARY 1 . Let $\mathscr{M}$ be the set of all functions $f \in C(X)$ such that $M(f)$ is dense in $X$. Then $\mathscr{M}$ is a dense subset of $C(X)$ endowed with the topology $\tau$.

Proof. This follows from the previous remark and the obvious fact that $\mathscr{M} \supset \mathscr{M}_{S}$ for every $\sigma$-discrete dense subset $S$ of $X$. The existence of such a set $S$ is deduced from the existence of a $\sigma$-discrete base for $X$. A more straightforward proof can be achieved as follows. For every $n=1,2, \ldots$ let $D_{n}$ be any subset of $X$ which is maximal with respect to the property: $x, y \in D_{n}, x \neq y \Rightarrow d(x, y) \geqslant 1 / n$; then $S=\bigcup_{n=1}^{\infty} D_{n}$ works.

We also have the following proposition which solves negatively Problem 3.2 of [2].

COROllary 2. Let the metric space $X$ be perfect. Then $\mathscr{R}(X)$ is a dense subset of $C(X)$ endowed with the topology $\tau$.

Proof. Since $X$ is perfect, we have $\mathscr{M} \subset \mathscr{R}(X)$. Then the density of $\mathscr{R}(X)$ follows from Corollary 1.

In view of the above corollary, we have that, for a metric space $X$, the perfectness of $X$ and the fact that $\mathscr{R}(X) \neq \varnothing$ are equivalent.

Finally, we would like to show how Theorem 1 enables us to solve positively Problem 3.1 of [2]; namely, whether the assumption of the local connectedness of $X$ can be dropped in Theorem A. As a matter of fact, Theorem 1 allows a further improvement of Theorem A-namely, obtaining the density of $\mathscr{R}(X, Y)$ in $C(X, Y)$ with respect to $\tau$.

THEOREM 2. Let $X$ be perfect. Then for every $g \in C(X, Y)$ and every $\varepsilon \in C(X)$, with $\varepsilon>0$ everywhere in $X$, there exists $g_{\varepsilon} \in \mathscr{R}(X, Y)$ such that $\left\|g(x)-g_{\varepsilon}(x)\right\|<\varepsilon(x)$ for each $x \in X$. Moreover, $g_{\varepsilon}(X) \subset \operatorname{conv}(g(X))$ provided that $g$ is not constant.

Proof. As it is not restrictive, we may assume $0 \in g(X)$ and $\varepsilon \leqslant 1$ everywhere in $X$. Let $\Sigma$ be any $\sigma$-discrete dense subset of $X$, and let $\Omega$ denote the set $\bigcup_{y \in Y} \operatorname{int} g^{-1}(y)$. Also, let $\eta \in C(X)$ be defined by $\eta(x)=\varepsilon(x) /(1+\|g(x)\|), x \in X$. By Theorem 1 
there exists $\lambda \in C(X)$ such that (i) $\lambda=-1$ in $X-\Omega$, (ii) $-1 \leqslant \lambda<-1+\eta$ everywhere in $X$, and (iii) $M(\lambda) \supset \Sigma \cap \Omega$. If $g$ is a constant, i.e, $g=0$ everywhere in $X$, we take $g_{\varepsilon}=(1+\lambda) \bar{y}$, with $\bar{y}$ any fixed element of $Y$ of norm one. If $g$ is not constant we fix $\bar{y} \in \operatorname{conv}(f(X))$, with $0<\|\bar{y}\| \leqslant 1$, and define $g_{\varepsilon}$ as follows: $g_{\varepsilon}(x)=-\lambda(x) g(x)$ if $x \in X-$ int $g^{-1}(0)$, and $g_{\varepsilon}(x)=(1+\lambda(x)) \bar{y}$ if $x \in$ int $g^{-1}(0)$. Then it is easy to check that $g_{\varepsilon}$ satisfies the thesis of the theorem.

\section{REFERENCES}

1. E. E. Posey and J. E. Vaughan, Functions with a proper local maximum in each interval, Amer. Math. Monthly 90 (1983), 281-282.

2. B. Ricceri and A. Villani, On continuous and locally non-constant functions, Boll. Un. Mat. Ital. A (6) 2 (1983), 171-177.

Dipartimento di Matematica, Citta Universitaria, Viale A. Doria 6, 95125 Catania, Italy 